\title{
Dust generation in powders: Effect of particle size distribution
}

\author{
Somik Chakravarty ${ }^{1, *}$, Olivier Le Bihan ${ }^{2},{\text { Marc } \text { Fischer }^{1} \text {, and Martin Morgeneyer }}^{1}$ \\ ${ }^{1}$ Université de Technologie de Compiègne (UTC) - Sorbonne Universités, Laboratoire Transformations intégrées de la matière \\ renouvelable (TIMR), Rue Roger Couttolenc, CS 60319, 60203 Compiegne Cedex, France \\ ${ }^{2}$ Institut National de l'EnviRonnement Industriel et des RisqueS (INERIS), NOVA/CARA/DRC/INERIS, Parc Technologique Alata, \\ BP2, F-60550 Verneuil-En-Halatte, France
}

\begin{abstract}
This study explores the relationship between the bulk and grain-scale properties of powders and dust generation. A vortex shaker dustiness tester was used to evaluate 8 calcium carbonate test powders with median particle sizes ranging from $2 \mu \mathrm{m}$ to $136 \mu \mathrm{m}$. Respirable aerosols released from the powder samples were characterised by their particle number and mass concentrations. All the powder samples were found to release respirable fractions of dust particles which end up decreasing with time. The variation of powder dustiness as a function of the particle size distribution was analysed for the powders, which were classified into three groups based on the fraction of particles within the respirable range. The trends we observe might be due to the interplay of several mechanisms like de-agglomeration and attrition and their relative importance.
\end{abstract}

\section{Introduction}

Granular matter or bulk solids makes up for roughly $50 \%$ of products and $75 \%$ of the raw material used in industrial applications [1]. Applications and processes involving handling or transportation of bulk solids generate dust, referred to as small solid particles which remain suspended in the air for a prolonged period of time [2]. The propensity of a material to generate dust upon handling is known as its dustiness [3]. The risks of dust emission in a contained area such as in an occupational environment can involve inhalation of dust particles by industrial workers [4,5] or explosion of volatile dust cloud capable of creating substantial financial and human loss [6]. Regulatory measures such as the 2008/50/EC directive [7] or ATEX [8] in the EU underpins the need for assessment and containment of dust concentration in ambient air with an emphasis on the generation and exposure of fine particles such as PM2.5 and respirable fraction, responsible for significant negative impacts on human health.

Dustiness of a material and thus the risk of exposure while handling a material depends on its physical properties and the type of process at work [9]. Testing of dust generation is often practical when developing new products in industries before producing and distributing them in bulk scale. Lab scale testing of dustiness of granular material requires a low-cost tester capable of testing a wide range of material with relatively simple operations. Standardized testers such as the continuous drop and the rotating drum method according to the EN $15051^{\prime}$ Workplace atmospheres-Measurement of the dustiness of bulk materials-Requirements and reference test methods' requires large amounts of powder $\left(35 \mathrm{~cm}^{3}\right.$ or $500 \mathrm{~g}$ ) and can give disparate results for a range of industrial minerals [4] due to the difference in stressing/agitation energy and the timescale of agitation, pointed out by several authors $[4,10]$.

New testers such as the vortex shaker (VS) enable testing dustiness of powders using a small fraction of the powder quantity required for the standardized testers [3]. They are especially suitable for testing micro- and nanoscale powders typically used in catalysts and pharmaceutical industries where the powder test quantity is low and costs are high. Furthermore, they are capable of testing powders for different energy levels by varying vortex speed and time. Morgeneyer et al. [3] and [11] used the VS method to study the effects of tester parameters on dust generation of micron-sized alumina particles and carbon nano-tubes (CNTs), respectively. In [3], the effect of the VS speed and sample mass on the dust generated from alumina were studied whereas [11] dealt with the effect of change in tube diameter in addition to vortex speed on dustiness of CNTs.

Measuring dustiness and the risk of exposure in different environmental conditions requires testing and characterizing powder properties by their effect on dustiness as it can enable understanding the role of different powder properties on the dust generation mechanisms. Since dustiness of a powder depends on several parameters, studying the effect of each physical parameter on dust generation requires testing the same material while changing one parameter at a time keeping the other powder parameters and environmental conditions constant.

\footnotetext{
* Corresponding author: somik.chakravarty@utc.fr
} 
In this study, respirable dustiness for eight calcium carbonate powders with similar physico-chemical properties were tested. The results were used to analyze the effect of particle size distribution (PSD) on the evolution of aerosol concentrations and size distributions. The powder samples were divided into three groups based on their PSD (and especially respirable fractions of particles already present in the powder) and attempted to identify 'potential scenarios' or generation mechanisms prevalent in each group.

\section{Material and experimental methods}

\subsection{Sample material}

The Eskal series of calcium carbonate powders (KSL Staubtechnik GmbH, Germany) were used as the test material. They are standard test powders used in various industries and also as a reference powder for calibration of equipment [12] due to their high roundness (close to 0.9 ), and insensitivity to moisture and temperature changes. All powder samples were manufactured with the same process/technology with the same particle density $\left(2,710 \mathrm{~kg} / \mathrm{m}^{3}\right)$, as reported by the manufacturer.

The volumetric size distribution of the powders (Table 1) were measured 'as received' using laser diffraction size analyser (Malvern Mastersizer 2000, Scirocco 2000M, UK). The samples were measured using dry dispersion of the powders with nozzle air pressure of 3 bars and the obscuration rate ranging from $1 \%$ to $5 \%$, depending on grain size of the samples.

Table 1. Volumetric size distribution of the tests samples.

\begin{tabular}{|c|c|c|c|c|}
\hline & Sample & $\begin{array}{c}\mathbf{X}_{\mathbf{1 0}}, \boldsymbol{\mu m} \\
(\mathbf{C O V}, \mathbf{\%})\end{array}$ & $\begin{array}{c}\mathbf{X}_{\mathbf{5 0}}, \boldsymbol{\mu m} \\
(\mathbf{C O V}, \mathbf{\%})\end{array}$ & $\begin{array}{c}\mathbf{X 9 0}, \boldsymbol{\mu m} \\
(\mathbf{C O V}, \%)\end{array}$ \\
\hline A1 & Eskal 300 & $0.96(3.3)$ & $2.2(3.1)$ & $4.6(3.4)$ \\
\hline A2 & Eskal 500 & $1.6(0.57)$ & $4.1(0.12)$ & $8.2(0.34)$ \\
\hline A3 & Eskal 1000 & $1.7(4.8)$ & $4.6(1.4)$ & $10(3.7)$ \\
\hline B1 & Eskal 10 & $5.7(1.2)$ & $10(1.1)$ & $16(1.2)$ \\
\hline B2 & Eskal 14 & $8.3(0.1)$ & $14(0.3)$ & $23(0.7)$ \\
\hline B3 & Eskal 15 & $8.8(0.3)$ & $16(0.13)$ & $25(0.11)$ \\
\hline B4 & Eskal 20 & $11(1.6)$ & $20(0.2)$ & $33(2.1)$ \\
\hline C1 & Eskal 150 & $99(0.5)$ & $136(0.1)$ & $187(0.5)$ \\
\hline
\end{tabular}

The primary selection criteria for the Eskal powders were their median particle size $\left(\mathrm{X}_{50}\right)$, their PSD and the respirable fraction of particles already present in the powder. The powder samples were classified in three groups with group A (A1, A2 and A3) consisting mainly of particles with size smaller than $10 \mu \mathrm{m}$, i.e., the maximum particle size sampled by a respirable cyclone. Group B (B1, B2, B3 and B4) consists of bi-modal powders, with modes at $(1.1 \mu \mathrm{m}, 11 \mu \mathrm{m}) \mathrm{B} 1,(1.9 \mu \mathrm{m}$,
$15 \mu \mathrm{m}) \mathrm{B} 2$, B3 $(2.2 \mu \mathrm{m}, 17 \mu \mathrm{m})$, and B4 $(2.9 \mu \mathrm{m}, 23 \mu \mathrm{m})$. Group C (C1) powder did not consist of particles in the respirable size range. Test samples from each group were designated by their group name followed by the sample number arranged in ascending order of their $\mathrm{X}_{50}$. For example, A1, A2 and A3 are the three samples from group $\mathrm{A}$ arranged in ascending order of their $\mathrm{X}_{50}$.

\subsection{The vortex shaker dustiness tester}

The experimental setup was similar to the one used by Morgeneyer et.al. [3] except that the released aerosol was sampled using a respirable cyclone (BGI GK2.69). The setup consists of 4 sections: generation, sampling, dilution, and measurement (Fig. 1). Aerosol is generated through the turbulent agitation of a powder-filled glass test-tube mounted on a digital vortex shaker (VWR Signature Digital Vortex Mixer).

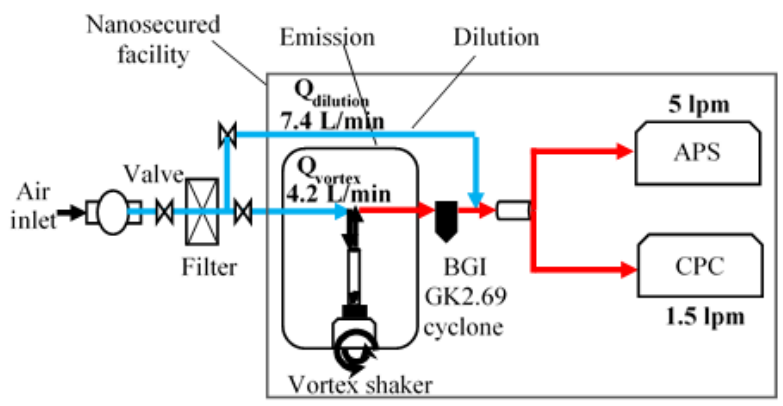

Fig. 1. Schematic of the vortex shaker experimental setup.

Airborne aerosol particles from the test-tube is carried to the respirable cyclone $(50 \%$ cut point of $4 \mu \mathrm{m})$ by the inlet flow $\left(Q_{v}\right)$ of $4.2 \mathrm{~L} / \mathrm{min}\left(7 \mathrm{e}-05 \mathrm{~m}^{3} / \mathrm{s}\right)$. While the cyclone separates the larger particles, the respirable aerosol particles permeates through the cyclone and is diluted with $\left(Q_{D}\right)$ of $7.4 \mathrm{~L} / \mathrm{min}\left(1.2 \mathrm{e}-04 \mathrm{~m}^{3} / \mathrm{s}\right)$ of filtered air before splitting into 2 channels for measurement and characterization [13]. The aerosol number concentration $N_{C P C}$ over the $4 \mathrm{~nm}$ to $3 \mu \mathrm{m}$ size range was measured using a condensation particle counter (CPC TSI 3775, TSI Inc.), whereas the aerosol number concentration $N_{A P S}$ and mass $M_{A P S}$ over the size range of $0.54 \mu \mathrm{m}$ to $20 \mu \mathrm{m}$ were measured by an aerodynamic particle sizer (APS TSI 3321, TSI Inc.). The APS records the particle numbers by their aerodynamic size which is based on times of flight of individual aerosol particles [3].

Three replicates were used for each test sample. Each test used $2 \mathrm{~g}$ of powder weighed with an accuracy of $\pm 0.001 \mathrm{~g}$ using an analytical balance (MS1003S, MettlerToledo Inc.), manually filled in a centrifuge glass tube (diameter $0.025 \mathrm{~m}$, height $0.15 \mathrm{~m}$ ). The filled tube was sealed using a rubber stopper and carried to the isolator system. The powders were weighed within 1 hour of performing the experiments to limit the number of variables affecting the powder condition. The VS operated at $1500 \mathrm{rpm}$ and was run for 10 minutes $(T)$. The background reference concentrations were measured for two minutes before the beginning and two minutes after the end of the vortex-shaker operation.

The total respirable aerosol number concentration measured by the CPC ( $4 \mathrm{~nm}$ to $3 \mu \mathrm{m})$ and the APS $(3 \mu \mathrm{m}$ 
to $19.5 \mu \mathrm{m}$ ) were combined to calculate the total number of generated particles $\mathrm{S}_{\text {Total }}^{\text {Number }}$ such as $\mathrm{S}_{\text {Total }}^{\text {Number }}=\mathrm{S}_{\mathrm{CPC}}^{\text {Number }}+\mathrm{S}_{\mathrm{APS}>3 \mu \mathrm{m}}^{\text {Number }}$ adapted from Jensen [13], whereby

$$
\begin{aligned}
& \mathrm{S}_{\mathrm{CPC}}^{\text {Number }}=\left[Q_{V}+Q_{D}\right] \cdot \Delta \mathrm{t}_{C P C} \cdot \sum_{\mathrm{i}=0}^{T / \Delta \mathrm{t}_{C P C}} N_{C P C}\left(\mathrm{t}_{0}+\mathrm{i} \cdot \Delta \mathrm{t}_{C P C}\right) \\
& \mathrm{S}_{\mathrm{APS}>3 \mu \mathrm{m}}^{\mathrm{Number}}=\left[Q_{V}+Q_{D}\right] \cdot \Delta \mathrm{t}_{A P S} \cdot \sum_{\mathrm{i}=0}^{\mathrm{T} / \Delta \mathrm{t}_{A P S}} N_{A P S>3 \mu m}\left(\mathrm{t}_{0}+\mathrm{i} \cdot \Delta \mathrm{t}_{A P S}\right)
\end{aligned}
$$

where, $\Delta \mathrm{t}_{\mathrm{CPC}}(1 \mathrm{~s})$ and $\Delta \mathrm{t}_{\mathrm{APS}}(5 \mathrm{~s})$ are the time-step set for the CPC and the APS, respectively. $\mathrm{dN}_{\mathrm{CPC}}\left(\mathrm{t}_{0}+\mathrm{i} . \Delta \mathrm{t}_{C P C}\right)$ and $d N_{A P S}\left(t_{0}+i . \Delta t_{A P S}\right)$ are the aerosol number concentration $\left(\# / \mathrm{cm}^{3}\right)$ for the $\mathrm{i}^{\text {th }}$ time interval measured by the CPC and the APS, respectively.

\section{Results and discussion}

In general, CPC and APS results reveal that all test samples emit respirable fractions of aerosol. The number concentration $\left(N_{C P C}\right)$ curves from CPC (Fig. 2) are the average values over 3 repetitions. Also, $\mathrm{S}_{\text {Total }}^{\text {Number }}$ calculated for the test samples are shown (Fig. 2, topright corner). All samples show an initial peak of $N_{C P C}$ at the onset of the VS activity (around 120s to 135 s from the start of the measurement). It is followed by a decline.
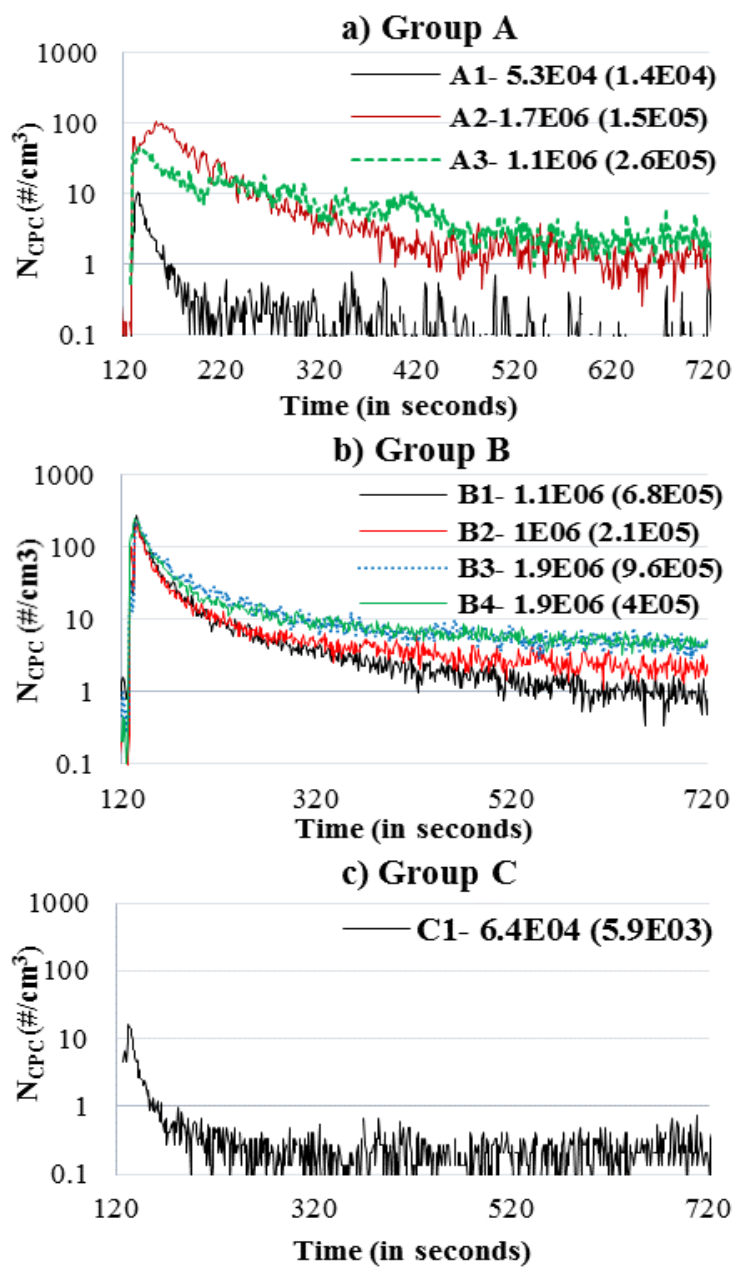

Fig. 2. Temporal evolution of respirable aerosol number concentration from the CPC for a) Group A; b) Group B; c) Group C. The total number of aerosol particles released $\mathrm{S}_{\text {Total }}^{\text {Number }}$ and their SD were calculated using (1) and (2) (see top-right corner).
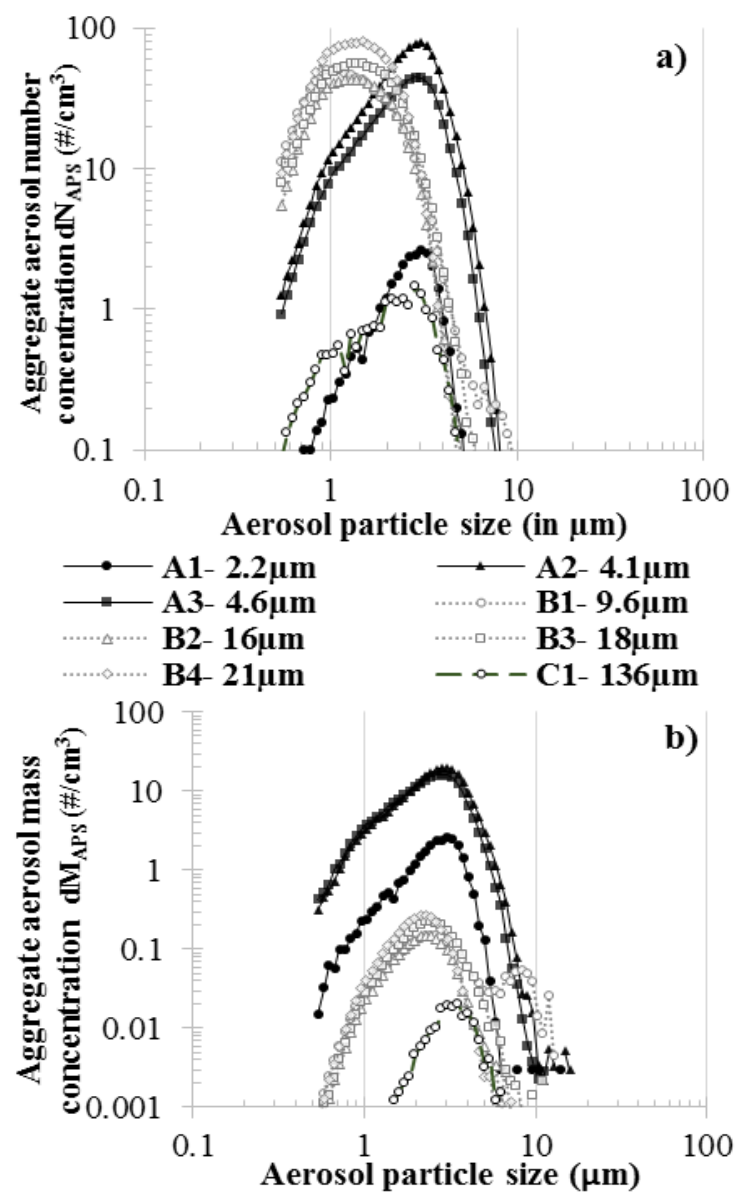

Fig. 3. APS measurements for aggregate aerosol concentration by particle size by a) number concentration $(\mathrm{dN})$; b) mass concentration $(\mathrm{dM})$.

\subsection{Group A}

Samples A2 and A3 show qualitatively similar dust generation behaviour, whereby they initially emit aerosols with concentrations up to $104 \# / \mathrm{cm}^{3}$ (A2) and $47 \# / \mathrm{cm}^{3}$ (A3) before a gradual decline. This stands in contrast to A1 which attains its maximum concentration of $10 \# / \mathrm{cm}^{3}$ before swiftly descending into emission of aerosols comparable to the background reference values. This relies on the assumption that the overwhelming majority of aerosol particles are in the size range measured by the CPC.

Measurements from the CPC (Fig. 2a) and APS (Fig. $3)$ shows the cumulative aerosol concentrations generated by $\mathrm{A} 2$ and $\mathrm{A} 3$ are greater than one order of magnitude higher than A1. Furthermore, the modal aerosol sizes of the three samples measured by the APS are similar and amount to approximately $3 \mu \mathrm{m}$ (Fig. 3).

Group A powders are cohesive in nature [14] and exist in the form of agglomerates of sizes greater than their primary particles. The dust generated from such powders could stem from the breaking and dispersion of the agglomerates into primary particles due to agglomerate-agglomerate collision or impacts against the wall. In the case of the VS, the centrifugal forces acting upon the agglomerates lead to collisions and impacts in the shaker which could result in the breakage and disintegration of agglomerates into particles. Such small 
disintegrated particles are more prone to be elutriated within a turbulent air flow developed in the VS. They follow a Stokesian flow regime [15].

A1 with a relatively small particle sizes (Table 1) forms agglomerates with high cohesive binding energies [14] such that the vortex agitation at $1,500 \mathrm{rpm}$ might not be enough to separate and aerosolize high concentrations of primary particles. Contrarily to A1, samples A2 and A3 consist of particles relatively larger in size and displaying broader PSD. Thus, based on the theoretical relationship between particle sizes and Van der Waals cohesive forces [16], the cohesivities of A2 and $\mathrm{A} 3$ must be inferior to that of A1.

For A2 and A3 (similar in size) the temporal evolution of the total number of released aerosols and their PSD are similar (Fig. 2 and Fig. 3). At the onset of vortex agitation, both samples emit relatively elevated concentrations of aerosols with small particle sizes. This might be due to the fact that such particles are more prone to be elutriated within the turbulent air flow [17].

\subsection{Group B}

The four bi-modal powders in group B display similar aerosol emissions during the test, whereby a sharp initial peak is followed by a gradual decline of the aerosol concentration into values between 1 and $10 \# / \mathrm{cm}^{3}$. B1 and $\mathrm{B} 2$ with the lowest $\mathrm{X}_{50}$ in this group emit relatively less respirable aerosols compared to B3 and B4. The modal aerosol size for all the samples measured from the APS is approximately $1 \mu \mathrm{m}$ (Fig. 3), which is in close proximity to the first mode particle size of B1 $(1.1 \mu \mathrm{m})$, B2 $(1.9 \mu \mathrm{m}), \mathrm{B} 3(2.2 \mu \mathrm{m})$, and B4 $(2.9 \mu \mathrm{m})$ (not shown). Thus the respirable fraction of aerosols from group B could originate from small particles with sizes inferior to the respirable size fraction.

Furthermore, laser diffraction measurements of volume fractions of particles with diameters smaller than $4 \mu \mathrm{m}$ present in group $\mathrm{B}$ shows that the respirable dustiness of a sample increases with an increase in the volume of particles in the first population. The volume fraction of the group B samples are measured as 3.7\% (B1), 4.9\% (B2), 5.2\% (B3), and 6.5\% (B4), with their mode particle size close $1 \mu \mathrm{m}$.

\subsection{Group C}

Group C represents powders with almost no particles within the respirable range. $\mathrm{C} 1$ initially releases an aerosol concentration peak at the onset of the VS followed by a rapid descent to aerosol concentration of 0 to $0.2 \# / \mathrm{cm}^{3}$, similar to background aerosol concentrations used as reference. The initial emission can be due to the presence of small impurities present in the powder or due to the generation of small fragments of powder due to the attrition of larger sized particles.

Several mechanisms can be responsible for the attrition of particles which depends on the particle mechanical properties, shape and mode of loading [18]. Large brittle particles are prone to generate dust by attrition as they contain more faults in the form of micro cracks or imperfections which can lead to fracture or breakage when compared to smaller particles.

An aerosol particle sampler, the mini-particlesampler (MPS $®)$ [13] could be used to capture and deposit aerosol particles on copper grids for off-site transmission electron microscope (TEM) analyses. This might provide evidence of the role of attrition in the generation of respirable aerosols.

\section{Conclusion}

The theoretical relationship between the properties of a powder and its dustiness remains poorly understood so that further experimental works are required $[3,11]$.

We use a vortex shaker to test respirable dust generation from eight powders with similar physical properties except their particle size distributions which differ from each other. The powders were divided into three groups based on the fraction of particles within the respirable range. The interplay of several mechanisms like de-agglomeration and attrition and their relative importance might account for our observations.

Further studies combining experimental (atomic force microscopy in micro-scale and shear test in mesoscale) and numerical (discrete element method) techniques are required in order to confirm this.

We acknowledge EU FP7 MCA ITN T-MAPPP and Région Picardie/ Hauts de France and by the Programme 190 (French Ministry of Environment) for their support.

\section{References}

1. R. Nedderman, Annals of Physics, 54 (2005)

2. Int. Org. Standardization, ISO 7708 (1995)

3. M. Morgeneyer, O. Le Bihan, A. Ustache, O. Aguerre-Chariol, Powder Technol. 246, 583 (2013)

4. I. Pensis, J. Mareels, D. Dahmann, D. Mark, Ann. Occup. Hyg. 54, 204 (2010)

5. C. Pujara, D. Kildsig, In J.P. Wood, editor., Drugs and the pharmaceutical sciences, 108 (2001)

6. A. Klippel, M. Schmidt, U. Krause, J. Loss Prev. Process Ind. 34, 22 (2015)

7. EU, Off. J. Eur. Communities., 152 (2008)

8. EU, Eur. Comm. Edition, 4 (2012)

9. M.A.E. Plinke, Am. Ind. Hyg. Assoc. J. 56, 251 (1995)

10. S. Bach, E. Schmidt, Ann. Occup. Hyg. 52, 717 (2008)

11. O.L.C. Le Bihan, A. Ustache, D. Bernard, O. Aguerre-Chariol, M. Morgeneyer, J. Nanomater., 7 (2014)

12. H. Zetzener, J. Schwedes, Part. Part. Syst. Charact. 19, 144 (2002)

13. K.A. Jensen, D4.6: EU NANOGENOTOX (2012)

14. H. Shi, S. Luding, V. Magnanimo, PGBSIA (2016)

15. S.C. Liang, T. Hong, L.S. Fan, Int. J. Multiph. Flow. 22, 285 (1996)

16. H. Rumpf, K. Sommer, K. Steier, Chemie Ing. Tech. 48, 300 (1976)

17. K. Saleh, M. Jaoude, M. Morgeneyer, E. Lefrancois, Powder Technol. 255, 141 (2014) 